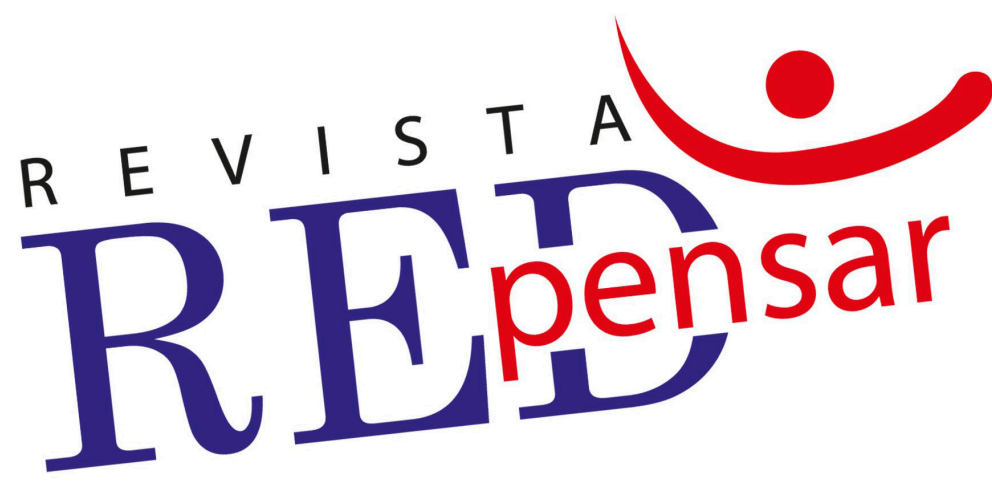

\title{
La Identidad:
}

\section{factor clave para la construcción de sociedad} Identity: key factor for the society construction

\section{Miguel Alejandro Barreto Crux}

Revista REDpensar, volumen 6, número 1, Ene-Jun 2017

ISSNe: 2215-5384

Lanzando la RED 1

Recibido: 30 de abril del 2018 Aceptado: 29 de junio del 2018

DOI: 10.31906/redpensar.v6i1.128

\section{(ㄷ)(1) $(2)$}




\section{La Identidad: factor clave para la construcción de sociedad Identity: key factor for the society construction}

Miguel Alejandro Barreto Cruz' ${ }^{1}$

\section{Resumen}

El presente artículo realiza una mirada al papel de las identidades de los sujetos como un elemento esencial para construir sociedad, teniendo en cuenta la importancia de estas desde un hecho histórico y su relevancia en cuanto al compromiso que tiene cada individuo con el espacio que le rodea y con aquellos con los que interactúa. Se plantea en este escrito la educación como una columna vertebral que puede fortalecer la identidad del sujeto como un aporte para la mejora de la sociedad. Se toman como autores invitados a este diálogo académico a Hannah Arendt y Walter Benjamin por su perspectiva de la violencia y la manera en cómo influye en la sociedad. Este texto tiene su origen en las reflexiones generadas en Seminarios de profundización del Doctorado en Ciencias de la Educación en la Universidad de San Buenaventura de Medellín, Colombia y deriva de discusiones académicas generadas en el marco de las investigaciones que se trabajan actualmente.

Palabras Clave: IDENTIDADES, EDUCACIÓN, SOCIEDAD, RELACIÓN CON EL OTRO, IDENTIDAD PERSONAL.

\section{Abstract}

This article looks at the role of the identities of individuals as an essential building society element, taking into account the importance of these from a historical event and its relevance to the commitment of each individual with the surrounding space and with those with whom it interacts. In this paper, education is considered as a backbone that can strengthen the identity of the subject as a contribution to the improvement of society. Hannah Arendt and Walter Benjamin are invited as authors of this academic dialogue for their perspective on violence and how it influences society. This text has its origin in the reflections generated in Seminars of deepening of the Doctorate in Sciences of the Education in the University of San Buenaventura of Medellín, Colombia and derives from academic discussions generated in the frame of the investigations that are worked at the moment.

Keywords: IDENTITY, EDUCATION, SOCIETY, RELATIONSHIP WITH THE OTHER, CULTURAL IDENTITY.

1. Administrador Financiero. Especialista en Gerencia de Instituciones Educativas. Docente líder de investigación, Facultad de Educación de la Corporación Universitaria Minuto de Dios - seccional Bello, Colombia. Magíster en Educación. Estudiante de Doctorado en Ciencias de la Educación, Universidad San Buenaventura - Medellín, Colombia. Correo electrónico: alejandrobarreto123@gmail.com 


\section{Introducción}

Si los hombres son seres del quehacer esto se debe a que su hacer es acción y reflexión. Es praxis. Es transformación del mundo. Y, por ello mismo, todo hacer del quehacer debe tener, necesariamente, una teoría que lo ilumine. El quehacer es teoría y práctica. Es reflexión y acción. No puede reducirse ni al verbalismo ni al activismo.

Paulo Freire (2005, p.161)

El propósito de esta reflexión se centra en reconocer el papel de la educación como una manera de influenciar las identidades de la comunidad estudiantil para conducirles hacia la transformación social, lo cual tiene como columna vertebral el reconocimiento de sí mismos, pero también la valoración del Otro como sujeto que puede aportar a la sociedad. Se plantea el asunto de las identidades, reconociendo su carácter dinámico, cambiante y teniendo en cuenta lo que expresa Bermejo (2011, p.46) "la identidad personal no parece estar fija [...] La persona se vivencia como si estuviera compuesta por múltiples seres, cada uno con [...] vida propia, yendo y viniendo sin atender la voluntad central de un yo único". No obstante, si bien se pone de manifiesto la forma mutable de las identidades, eso no significa la ausencia de características en los sujetos, que configuren la forma de ser y de actuar en el mundo, y que evolucione con el paso del tiempo. Por ende, en este artículo se propone considerar el cómo la identidad ha sido atacada en la historia, y el papel que ésta juega para pensar en la transformación de la sociedad teniendo como base una propuesta educativa.

Es así como se plantea que la sociedad y sus problemáticas requieren una mirada desde la educación, porque se espera que desde ella misma surjan propuestas que lleven a una transformación social. Ahora bien, no se puede pensar en sociedad sin primero realizar una mirada profunda sobre los individuos que la componen y la forma de relacionarse con Otros en espacios como los educativos, es en este punto que surgen varias interrogantes, ¿por qué es relevante hacer una mirada a la sociedad desde las identidades de cada sujeto?, ¿los problemas sociales tienen su origen en las problemáticas de la identidad?, ¿qué papel juega la educación sobre la identidad como una constructora de la sociedad?

Las ideas plasmadas en este texto forman parte del proceso doctoral en Ciencias de la Educación, el cual promueve el diálogo académico sobre la relevancia que tienen las identidades desde la educación, de esta manera, dejar una provocación para quien quiera estudiar la triada: identidades - sociedad - educación. Por ende, es relevante en primera instancia establecer una mirada para comprender la relación existente entre lo que son las identidades y la sociedad.

\section{Una mirada a la relación identidades y sociedad}

Lo complejo de abordar las problemáticas sociales y el tratar de comprender la sociedad, radica en que está compuesta por seres humanos diferentes, únicos e irrepetibles, y así como cada sujeto físicamente cuenta con una huella digital que lo identifica entre más de siete mil millones de personas que viven hoy sobre el planeta tierra, y que ninguna otra persona puede tener, lo hace un ser único, diferente e irrepetible. La identidad es un factor o la esencia para poder pensar la sociedad y construirla no hay sociedad sin sujetos y los sujetos no pueden existir si no están en el marco de una sociedad, tal y como lo afirma Mélich (2010) cuando partiendo de la moral expresa cómo debemos comportarnos en el mundo y fija una serie de comportamientos, actitudes y reglas que debemos seguir las cuales están dadas desde que nacemos y termina afirmando que esa moral configura nuestra identidad haciendo además una precisión relevante al decir "toda identidad es social" (p.327).

Ahora bien, la consideración de la identidad hace parte de un asunto trascendental para el Ser Humano y ha sido un tema que se ha estudiado por siglos desde diferentes áreas, la misma autora señala "Kant sostuvo que todas las preguntas filosóficas podían resumirse en una: ¿qué es el hombre? La cuestión antropológica, la cuestión por el ser de la realidad humana, es, según él, la fundamental" (Mélich, 
2010, p.327). No obstante, es importante hacer un acercamiento a las concepciones que se tiene de las identidades, puesto que su definición puede variar si se hace una mirada desde la antropología, la filosofía, la psicología u otra área del conocimiento. Mortimer, Wortham y Allard (2010) señalan "las identidades, están en movimiento, múltiples y heterogéneamente evaluadas. Como los signos y modelos de identidad se mueven más rápido y más extensamente en nuestra era globalizada, el proceso de identificación social se ha vuelto más complejo" (p.112).

Es importante precisar que para este artículo se asume una postura desde la psicología educativa, donde se reafirma que "la identidad es asumida como un proceso de construcción abierto de manera permanente, no sólo a través de la historia del individuo sino también cambiante en el devenir histórico de la sociedad" (Monereo y Pozo, 2011, p.195). Empero, surgen preguntas como ¿si las identidades son cambiantes, entonces para qué hablar de construirla?, ¿si las identidades varían según la historia, entonces se puede pensar en preservarla? En este sentido, es importante tener presente que dentro de las diferentes gamas de las identidades que existen como la social, la cultural, entre otras, se puede hablar de la identidad personal. Monereo y Pozo (2011) indican,

La utilidad de la identidad es ofrecer una estructura de continuidad a nuestra existencia (Quién soy, cómo soy, a qué aspiro en la vida, etc.) que ordene posibles versiones de "sí mismo" en competición y permita sabernos la misma persona y, a la vez, reconocernos como cambiantes a través del tiempo. (p.63)

Ahora bien, la necesidad de atender la identidad, es porque en ella misma radica la posibilidad de construir o destruir la sociedad, ya que "el sentido de identidad puede ser fuente no sólo de orgullo y alegría, sino también de fuerza y confianza [...] Y, sin embargo, la identidad también puede matar, y matar desenfrenadamente" (Sen, 2007, p.23). De esta manera, se hace evidente que sin identidad, existe el riesgo del surgimiento de una descomposición social, y por eso, hay una relación estrecha que no puede ser pasada por alto y es que "es en los procesos de socialización dónde y cuándo el sujeto social construye su identidad y, que ésta es definida en el conjunto mismo de las relaciones que el individuo mantiene con los demás" (Monereo y Pozo, 2011, p.193). De ahí que es imperioso hacer una mirada a este importante tema y considerarlo desde diferentes ámbitos en los cuales tiene una incidencia directa y conocer sobre los aspectos relevantes que la influencian y su vital importancia, teniendo como ejemplo los ataques que han existido contra la identidad en la historia.

\section{Ataques contra la identidad en la historia}

A partir de la afirmación de las identidades ligadas al contexto, una mirada importante es la de la historia, específicamente la manera en cómo la identidad ha sido blanco de ataques en los conflictos bélicos, en este caso nos referimos a la Segunda Guerra Mundial. En el caso de los judíos que estuvieron al borde del exterminio por parte de los nazis, si bien, existió un exterminio físico de millones de personas, el mayor ataque de los nazis hacia los judíos fue borrarles su identidad, arrancarles su historia, su memoria, hasta borrarles sus nombres y cambiarlos por un número. Con la humillación, el miedo, la muerte y el sufrimiento buscaron borrar la esencia de todo lo que les hacía humanos y lo que les constituía como personas, su identidad. Una descripción de este fatídico suceso es lo descrito por David Rousset (1947) (citado por Arendt, 1999) en su relato sobre la realidad de los campos de concentración nazi,

El triunfo de las SS exigía que las víctimas torturadas se dejaran conducir a la horca sin protestar, que renunciaran a todo hasta el punto de dejar de afirmar su propia identidad. Y esta exigencia no era gratuita. No se debía a capricho o a simple sadismo. Los hombres de las SS sabían que el sistema que logra destruir a su víctima antes de que suba al patíbulo es el mejor, desde todos los puntos de vista, para mantener a un pueblo en la esclavitud, en total sumisión. Nada hay más terrible que aquellas procesiones avanzando como muñecos hacia la muerte. (Arendt, 1999, p.12)

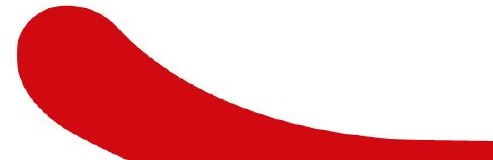


Es evidente la existencia de un arma poderosa contra la humanidad, que destruye el cuerpo o más potente que una bomba atómica, y es el arma que atenta contra la identidad, puesto que al despojar a un sujeto de su identidad, lo convierte en un muerto en vida, hace del sujeto como un animal que camina hacia el matadero, que pierde el impulso para luchar, para pensar, para reflexionar y por ende para vivir.

Es innegable que en todo conflicto bélico siempre hay víctimas y son muchas las personas inocentes que pierden sus vidas, puesto que, los seres humanos son utilizados como escudos, menospreciando el valor de sus vidas y otorgando mayor relevancia a los intereses políticos, económicos y hasta personales. Butler (2014, p. 106) afirma que, "toda vida humana ha sido instrumentalizada como parte de la guerra y, como resultado, ha dejado de ser una vida que vale la pena proteger, precaria, carente , una vida que vale la pena apreciar y lamentar."

El valor por la vida es un imperativo ético para toda sociedad, pero sobretodo es necesario plantear el valor por el individuo, el valor de la identidad de cada sujeto, y en ese sentido se requiere de gobiernos, instituciones y movimientos sociales. Cuando se impone una ideología y se considera que es la única verdad, se cae en desgracias como la que tuvieron que enfrentarse los judíos, a una corriente que se consideraba una raza superior, y de esa manera afrontaron un ataque contra su identidad, con repercusiones más allá de lo físico, cuando se plantearon ideologías que atentaban contra su propia identidad, por eso tal y como lo señala Mate (2009, p. 112) se puede decir que "hasta en la historia de la filosofía puede uno constatar que hubo un ajusticiamiento metafísico del judío mucho antes de que tuviera lugar el exterminio físico." Por tanto, toda transformación social debe ser guiada bajo los principios del respeto por la identidad y debe considerarse la identidad como una posibilidad de aprendizaje y no como una amenaza que requiere ser eliminada.

\section{Generando transformación social desde la identidad de cada sujeto}

Los principios que constituyen la formación del sujeto, requieren la intencionalidad de brindarle herramientas que le permitan la posibilidad de tomar decisiones responsables, de actuar de manera coherente, de una u otra manera lograr que cada sujeto asuma el rol que le corresponde, puesto que si cada individuo, conoce su papel en el mundo y tiene claro que sus acciones tienen consecuencias, buscará la manera de vivir coherentemente. Una conclusión extraordinaria frente a las actuaciones que tenemos como seres humanos es la que aporta Berstein (2015) donde realiza un análisis de la violencia desde la perspectiva de Walter Benjamin, quien afirma que "lo único de lo que podemos estar seguros es que debemos asumir con honestidad la responsabilidad por nuestros actos" (p. 89), y es así, como es posible enmarcar que, las acciones están sujetas a nuestra identidad y la manera de encaminar esas acciones que propendan por la transformación social es precisamente fortaleciendo la identidad de cada sujeto.

En cierta medida, es complejo plantear como punto de partida al individuo para buscar un cambio o una transformación, especialmente si se piensa en una globalidad o un impacto a gran escala, pero es necesario reconocer que por inmensa que sea la humanidad, los verdaderos cambios a nivel social no pueden desconocer la particularidad de cada sujeto y desde ahí establecer acciones para un verdadero cambio, puesto que toda sociedad está compuesta por individuos, y es la agrupación de esos individuos la que finalmente conforman un grupo social. Aunque el tema central aquí es la necesidad del individuo, conviene tener en cuenta lo expresado por Hillesum (2007) citada por Mate (2009), “[...] no se puede cambiar el mundo si antes no cambia el corazón y la mente de cada individuo" (p.122). Se requiere entonces de una sinergia que empieza desde una identidad propia y sana, que trasciende para relacionarse adecuadamente con los demás, tal y como se ilustra en el siguiente gráfico. 
ILUSTRACIÓN 1.

Interaciones de la identidad

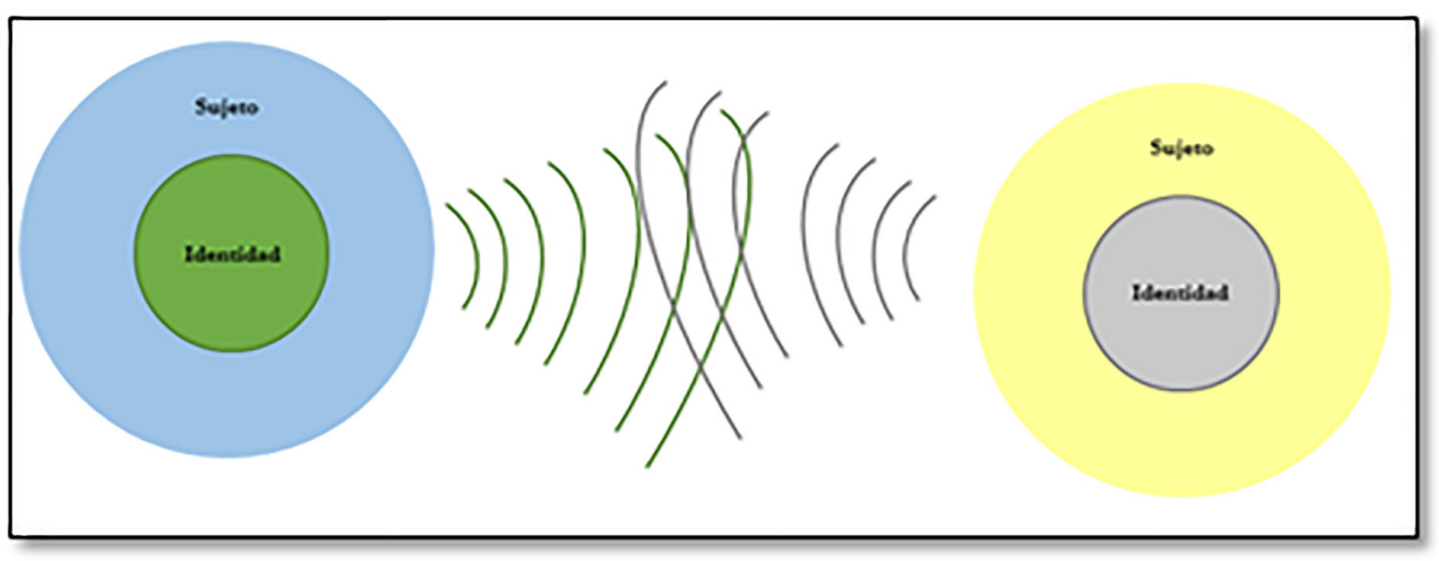

Fuente: Elaboración propia, 2018.

Es así como se requiere despertar la consciencia de cada sujeto, para que realice una contribución positiva a la sociedad y eso es posible únicamente protegiendo y fortaleciendo la identidad de los sujetos, entendiendo que esta "es un sistema de percepciones y actitudes de la persona hacia sí misma. La identidad, por lo tanto, es parte de la conciencia del individuo" (Bradley, 1998). Por ende, si una persona no tiene consciencia de sí misma, tampoco puede ser consciente del Otro y tener capacidad de comprenderle, respetarle y valorarle su existencia, teniendo en cuenta el principio de Bermejo (2011) "la identidad, tanto la colectiva como la individual, cumple unas funciones importantes para el ajuste social y el bienestar de las personas" (p.256).

\section{La identidad propia y su relación con el Otro}

Si bien, ya se han puesto en discusión las identidades desde un marco social, existen algunas precisiones puntuales que valen la pena distinguir, las relaciones del sujeto con el Otro y desde una óptica de identidad. Si bien se trata de algo meramente personal, continúa siendo claro que como seres humanos somos sociales por naturaleza y también desarrollamos una identidad social, como bien lo señala Sen (2007, p.24) "el sentido de identidad puede contribuir en gran medida a la firmeza y la calidez de nuestras relaciones con Otros, como los vecinos, los miembros de la misma comunidad, los conciudadanos o los creyentes de una misma religión".

El proceso de la identidad involucra un reconocimiento del Otro, ya que desde esta manera se evita el individualismo y una visión egoísta de la vida. El Yo es importante, requiere ser considerado y fortalecido para la propia identidad del Ser Humano, sin embargo, si la identidad no es construida con una visión responsable hacia el Otro puede resultar peligrosa. Desde un marco de identidad cultural Sánchez, Aguirre y Ochoa (2015) resaltan la escuela como un escenario para la formación del individuo, por ser un espacio de interacción y además un lugar para conocer otras culturas, y en esa medida cuando se logra consolidar una identidad cultural, se evita que la persona tenga una perspectiva que no valora a los demás, que es egocéntrica y que finalmente como lo señalan los autores "se convertiría en un problema más para la sociedad actual que de lo único que está ávida en estos momentos es de personas con valores y principios” (p.73).

De esta manera, es suficientemente evidente que las identidades son un tema crucial para la comprensión de la sociedad y esa identidad social puede ser un obstáculo o un propulsor para la interacción y convivencia humana. Merino (2015) expresa que "no hay duda de que el poder que confiere la identidad [...] para reconocerse dentro de una comunidad determinada, permite que se siga insistiendo en cuáles son los rasgos

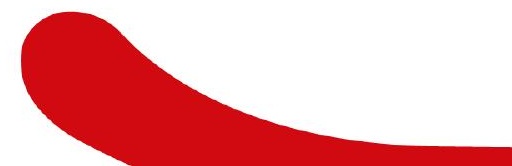


que configuran y determinan la identidad en el sujeto" (p.11). Un ser humano sin identidad, es una persona que no tiene la capacidad de relacionarse con Otros, peor aún, un ser humano con una identidad distorsionada puede dañar a Otros, la identidad tiene la capacidad de impulsar nuestras actuaciones, al respecto Merino (2015) nos señala

Desde tiempos inmemoriales el ser humano ha estado forjando su historia o reconquistando el poder por medio de la identidad. ¿Cómo se explica el masivo y popular seguimiento de Hitler para emprender su proyecto político? ¿Por qué Mandela fue capaz de unificar una nación profundamente dividida? ¿Qué ha permitido que grupos terroristas, en nombre del Islam, sean capaces de terminar con la vida de mucha gente inocente? (p.11)

Los anteriores son ejemplos claros expuestos por Merino en su tesis doctoral ${ }^{2}$, donde expone la manera en que la identidad ha tenido un papel protagónico en la historia de la humanidad y la forma en cómo los seres humanos nos hemos organizado, relacionado y hasta enfrentado en el tiempo. Entonces, si la identidad juega un papel tan trascendental, ¿por qué no darle un lugar prominente en la educación?

Este es el gran desafío, considerando que la educación tiene un papel central en la vida del Ser Humano. Tiene la posibilidad de influenciar a toda la sociedad, ya que por sus aulas pasan todas las profesiones, todos los cargos gubernamentales, todos los que tienen el poder económico y todos lo que componen la sociedad independientemente de su papel en la escala social, siendo una gran responsabilidad y tarea para quienes asumen el papel en la educación.

Ahora bien, más que pensar en homogeneizar dando determinados contenidos, se requiere la habilidad de influenciar la identidad de cada sujeto, reconociendo su individualidad y particularidad. Esta es la invitación que hace la autora Rosa Blanco (2008) en su exposición sobre Educación Inclusiva ${ }^{3}$ cuando se le adjudica al sistema educativo la responsabilidad de brindar una educación que tome en cuenta la diversidad, al enfatizar sobre la necesidad de "modelos educativos que consideren la diversidad de necesidades, capacidades e identidades [...] de forma que la educación sea pertinente para todas las personas y no sólo para determinados grupos de la sociedad" (p.8).

Empero la pregunta central que queda planteada es ¿cómo construir sociedad desde la identidad?, y la respuesta implica algo tan complejo como el Ser Humano mismo, pero, una pista para entender esta complejidad y a la vez encontrar una respuesta está en la consciencia que necesita tener cada sujeto de sí mismo y de su realidad. Finalmente la respuesta a la crisis social, a la violencia, a la maldad, a la destrucción del propio ser humano, está en lo que constituye la identidad de cada sujeto, como lo es el corazón del Ser Humano, las relaciones con Otros, el amor y la confianza. Una tesis central de este enfoque de consciencia está en la reflexión que hace Benjamin (2010) en su ensayo «Hacia la crítica de la violencia), con una de sus reflexiones centrales, la cual es una evidencia de lo que involucra una consciencia de la identidad y por ende, confiere un aporte relevante para este texto.

¿Es posible resolver los conflictos sin violencia alguna? Sin duda que sí: las relaciones privadas entre personas están llenas de ejemplos de esto. El acuerdo carente de violencia se encuentra donde la cultura del corazón ha puesto a disposición de los hombres medios puros de acuerdo. Hay que contraponer como medios puros a los legales e ilegales de cualquier tipo (todos ellos violentos sin excepción) aquellos otros medios que carecen de violencia. Así la cortesía del corazón, la inclinación y el amor hacia la paz, junto a la confianza y otras cosas que aún podríamos nombrar, son su presupuesto subjetivo." (p.195)

2. Cuyo título es "El desarrollo de la identidad docente en el profesorado de educación secundaria en un nuevo contexto social. Un enfoque biográfico-narrativo”, desarrollada en el 2015.

3. Administrador Financiero. Especialista en Gerencia de Instituciones Educativas. Docente líder de investigación, Facultad de Educación de la Corporación Universitaria Minuto de Dios - seccional Bello, Colombia. Magíster en Educación. Estudiante de Doctorado en Ciencias de la Educación, Universidad San Buenaventura - Medellín, Colombia. Correo electrónico: alejandrobarreto123@gmail.com 


\section{El aporte de la educación a la identidad}

La educación ha sido y siempre será un factor clave y fundamental para el desarrollo de cualquier sociedad y por eso, vale la pena considerar la manera en que ésta realiza un aporte significativo a los sujetos para la construcción de una identidad que lleve a pensar en una mejor sociedad, tal y como lo señalan Monereo y Pozo (2011, p.37) "la comprensión de la identidad no sólo pasa por responder a la vieja pregunta acerca de si, “¿quién soy yo?”, sino a otros proyectadas a un futuro más o menos lejano: “¿qué quiero ser?”, "¿qué puedo hacer?" ". Definitivamente, el pensar en qué puede convertirse un Ser Humano y qué es capaz de hacer es una asunto que se aborda de manera efectiva desde la educación, teniendo presente que en la escuela se pretende formar un ideal de persona y se intenta darle herramientas al sujeto para que éste se desenvuelva de una manera más efectiva en la sociedad. Como lo indica Bermejo (2011),

La praxis de relaciones interpersonales constitutivas de la identidad se complementa desde el conjunto de las instituciones sociales, que juegan un papel determinante en el proceso de formación de la identidad personal, de la integración del individuo en la sociedad, y de la elaboración de un proyecto de vida con sentido. (p.302)

Así las cosas, el ámbito educativo se convierte en una base fundamental sobre la cual se ubican los individuos y a través de ellos se permea el escenario social. Por tanto, surge la necesidad que las personas profesionales en docencia tengan una consciencia despierta en cuanto a las características de la sociedad y a la vez las características de sus estudiantes, para encontrar la manera de potenciar la influencia que se tiene sobre lo social; aunque es importante aclarar que si bien la educación es crucial, no recae sobre ella toda la responsabilidad, puesto que, "la construcción de una identidad múltiple, enriquecedora y no conflictiva, no es una tarea fácil porque, entre otras cosas, hace falta una mayor continuidad educativa entre la familia, la escuela y la comunidad" (Coll, 2000; Vila y Casares, 2009, citados por Vila, Guitart y Oller, 2010,p.63).

No obstante, el sistema educativo tendrá mayor oportunidad en sus manos, al influir directamente sobre muchas generaciones, Monereo y Pozo (2011, p.112) mencionan el hecho que "los aprendices van construyendo una identidad al aprender contenidos significativos", y esa oportunidad es la responsabilidad de ofrecer contenidos que respondan a las necesidades, expectativas y realidades de sus estudiantes, puesto que en la medida que logran conectar lo aprendido en el aula con lo que viven en las calles y en sus casas, logran cosntruir y afianzar la identidad del estudiante en ese proceso aprendizaje.

Lo anterior se asimila a los retos y cambios que el mundo afronta constantemente, lo que también muestra es un sistema educativo que está en constante actualización, pero que no puede desligarse del contexto, a esto se refieren Monereo y Pozo (2011) cuando frente a ese contexto cambiante afirman "la educación, la escuela y el profesorado no pueden mantenerse al margen de este conjunto de situaciones. La escuela, porque históricamente ha tenido la misión de crear identidades sociales y culturales ajustadas a las demandas procedentes del contexto [...]" (p.190).

Una manera de visualizar esto es mediante la siguiente ilustración que plantea la identidad del sujeto como centro, quien recibe la influencia de diferentes contextos con los cuales se relaciona y diáloga con ellos. Se trata de una relación de influencia mutua y que si bien puede representar tensiones, el caso aquí es la necesidad de reconocer algunos de los muchos escenarios de los cuales hace parte el Ser Humano, entre los cuales está la educación, que si bien tiene un papel preponderante no es la única que tiene ingerencia sobre la identidad y por eso es necesario contemplar todas las influencias existentes.

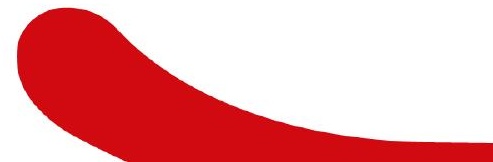




\section{ILUSTRACIÓN 2. \\ Contextos que inciden en la identidad del sujeto}

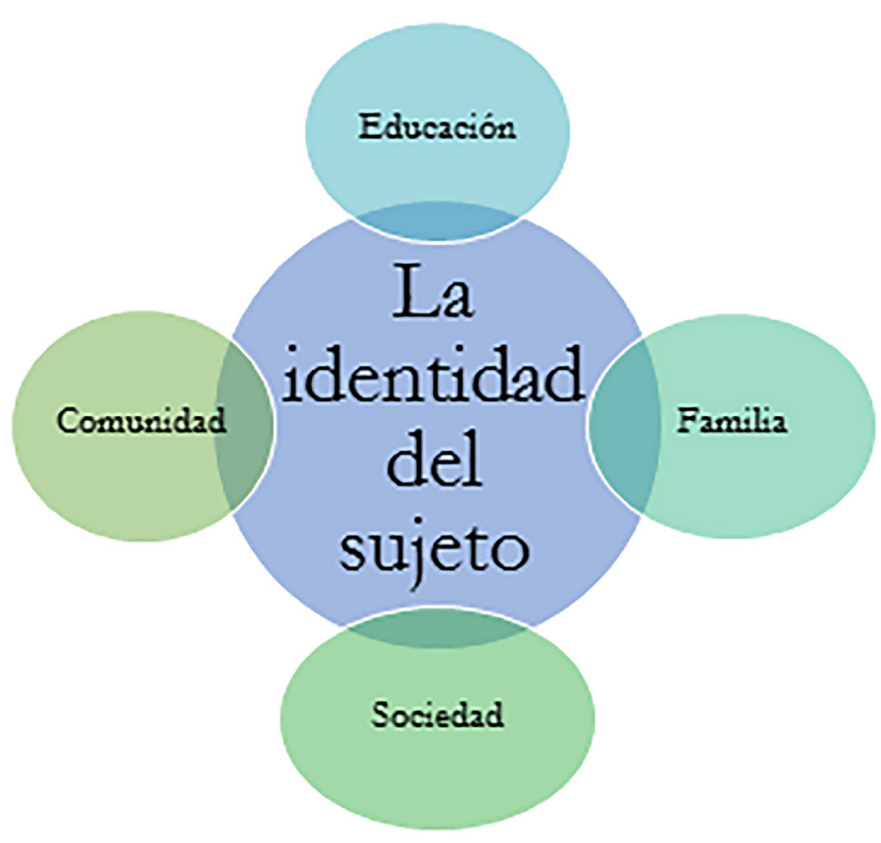

Fuente: Elaboración propia, 2018.

De esta manera, desde la educación se requiere contruir puentes entre los saberes cotidianos, las preguntas que emergen de la vivencia diaria y a su vez lo que la academia tiene para decir; ese diálogo se da a partir de los actores involucrados en el ámbito educativo: estudiantes y docentes, quienes construyen su identidad influenciados por diferentes escenarios como la escuela, al partir de la premisa indicada por los autores Monereo y Pozo (2011, p.81) "el concepto de identidad engloba el conjunto de procesos dinámicos y dialógicos que hacen posible el movimiento de las personas entre diferentes contextos que conforman su mundo, así como su participación y su actividad en estos contextos"; por ello, no se puede desconocer que si bien la educación aborda ese basto mundo del conocimiento, ella no lo abarca todo, sino que sólo tiene una parte y no debe desconocer aquellas otras fuentes como las de la vida misma, puesto que todo esto influye sobre la identidad, de ahí que "el saber cotidiano es condición indispensable para la sociabilidad y la comunicación de los sujetos; esto es el mundo intersubjetivo inmediato, donde se comparte con otros: memoria, territorio, identidad y sentidos" (Ghiso, 2015, p.29). Así las cosas, si la educación quiere ser pertinente en la construcción de identidad requiere recuperar en sus aulas el valor del saber cotidiano..

\section{Conclusiones}

La historia evidencia que hay un aspecto metafísico que da sentido a lo que es el Ser Humano y es precisamente la identidad personal, por lo que atacar la identidad significa un atentado contra la humanidad. Es por esto, que vale la pena la consideración de la identidad como una manera de preservar la sociedad y a su vez hacer un aporte para construirla.

En línea con lo anterior, se debe partir entonces de preservar la identidad de cada sujeto y lo que es éste, como una manera de contribuir a las buenas relaciones sociales, debido a que, en la interacción con Otros se construye la identidad personal, la cual, si está bien orientada lleva a relacionarse de manera 
correcta con Otros, generando una sinergia o conexión que se traduce en una mejor sociedad.

No obstante, si bien se puede hablar de la existencia de una identidad personal, también es necesario comprender la existencia de diversas identidades que pueden enmarcarse en lo cultural, en lo social y en los diferentes espacios y contextos en los que desenvuelva el sujeto. Además, si bien las identidades se reconocen con identidades dinámicas y cambiantes, eso no implica que no tengan características propias, que puedan ser analizadas.

Finalmente, en todo este escenario se hace claro que la educación juega un papel crucial para la construcción de la identidad de los sujetos, en la medida que hace un aporte desde los aprendizajes que se socializan en el aula, si es que estos son significativos al conectar los contenidos con la realidad. Si se tiene una educación aterrizada al contexto, entonces el individuo potencializará su identidad y a la vez hará un aporte significativo a la sociedad.

\section{Referencias Bibliográficas}

Arendt, H. (1999). Eichmann en Jerusalén. Un estudio sobre la banalidad del mal. Barcelona, España: Editorial Lumen S.A.

Benjamin, W. (2010). Hacia una crítica de la violencia. Madrid, España: Editorial Abada.

Bermejo, D. (2011). La identidad en sociedades plurales. Barcelona, España: Editorial Anthropos.

Berstein, R. (2015). Violencia: Pensar sin barandillas. Barcelona, España: Editorial Gedisa.

Bradley, R. (1998). Identidad judia: Una construcción de identidad negativa vista en el contexto de la Diáspora. Buenos Aires, Argentina: II Jornadas de Medio Oriente, Departamento de Medio Oriente. Facultad de Ciencias Jurídicas y Sociales, Universidad Nacional de la Plata [UNLP]. Recuperado de https://bit.ly/2uqksj0

Butler, J. (2014). ¿A quién le pertenece Kafkea? Chile: Editorial Palinodia.

Coll, C. (2000). Educación, territorio y responsabilidad ciudadana. En J. A. Garde (Ed.), Informe 2000. Politicas sociales y estado de bienestar en España (pp. 165-187). Madrid, España: Fundación Hogar del Empleado.

Freire, P. (2005). Pedagogía del oprimido (2da ed.). México: Editorial Siglo XXI.

Ghiso, A. M. (2015). Del diálogo de saberes a la negociación cultural. Recuperar, deconstruir, resignificar y recrear saberes. Revista REDpensar, 4(1), 27-39.

Hillesum, E. (2007). Diario de Etty Hillesum. Una vida conmocionada. Madrid, España: Anthropos Editorial.

Mate, R. (2009). La berencia del olvido. Madrid, España: Editorial Errata Naturae.

Mèlich, J.C. (2010). Poética de lo íntimo (sobre ética y antropología). Ars Brevis: anuario de la Càtedra Ramon Llull Blanquerna, (16), 314-331.

Merino, M. (2015). El desarrollo de la identidad docente en el profesorado de educación secundaria en un nuevo contexto social. un enfoque biográfico-narrativo. Sevilla, España: Universidad de Sevilla.

Monereo, C., y Pozo, J. I. (2011). La identidad en psicología de la educación: necesidad, utilidad y limites. Madrid, España: Narcea Ediciones. 
Mortimer, K., Wortham, S., y Allard, E. (2010). Helping immigrants identify as «university-bound students»: unexpected difficulties in teaching the hidden curriculum. Revista de Educación, (353), 107-128.

Blanco, R. (2008). La Educación Inclusiva: El camino hacia el futuro. En R. Blanco (Directora Interina), Conferencia Internacional de Educación. Conferencia llevada a cabo en la Cuadragésima Octava Reunión Centro Internacional de Conferencias, Ginebra, Suiza. Recuperado de https://bit. ly/1vEFmCj

Sánchez, I. M., Aguirre, W. R., y Ochoa, J. C. (2015). La identidad cultural como elemento clave para profundizar en los procesos educativos que apunten a la convivencia en sociedades multiculturales. Praxis, 11, 61-75. doi:10.21676/23897856.1554

Sen, A. (2007). Identidad y violencia. La ilusión del destino. Buenos Aires: Katz Editores.

Vila, I. y Casares, R. (2009). Educación y sociedad. Una perspectiva sobre las relaciones entre la escuela y el entorno social. Barcelona, España: Editorial Horsori.

Vila, I., Guitart, M., y Oller, J. (2010). Identidad nacional, lengua y escuela. Revista de educación, (353), 39-65. 